\title{
WHO Grade IV Gliofibroma: A Grading Label Denoting Malignancy for an Otherwise Commonly Misinterpreted Neoplasm
}

\author{
Paola A. Escalante Abril \\ Miguel Fdo. Salazar \\ Nubia L. López García \\ Mónica N. Madrazo Moya \\ Yadir U. Zamora Guerra \\ Yadira Gandhi Mata Mendoza \\ Erick Gómez Apo \\ Laura G. Chávez Macías \\ Pathology Unit, Neuropathology Service, \\ Mexico General Hospital, Mexico City, Mexico \\ Received: March 2, 2015 \\ Revised: May 10, 2015 \\ Accepted: May 20, 2015 \\ Corresponding Author \\ Miguel Fdo. Salazar, MD \\ Hospital General de México "Dr. Eduardo Liceaga", \\ Unidad de Patología, Servicio de Neuropatología, \\ Dr. Balmis 148, Col. Doctores, Delegación \\ Cuauhtémoc, C.P. 06726, México D.F. \\ Tel: +52-55-01-55-2789-2000 \\ E-mail:k7nigricans@hotmail.com
}

\begin{abstract}
We report a 50-year-old woman with no relevant clinical history who presented with headache and loss of memory. Magnetic resonance imaging showed a left parieto-temporal mass with annular enhancement after contrast media administration, rendering a radiological diagnosis of high-grade astrocytic neoplasm. Tumour sampling was performed but the patient ultimately died as a result of disease. Microscopically, the lesion had areas of glioblastoma mixed with a benign mesenchymal constituent; the former showed hypercellularity, endothelial proliferation, high mitotic activity and necrosis, while the latter showed fascicles of long spindle cells surrounded by collagen and reticulin fibers. With approximately 40 previously reported cases, gliofibroma is a rare neoplasm defined as either glio-desmoplastic or glial/benign mesenchymal. As shown in our case, its prognosis is apparently determined by the degree of anaplasia of the glial component.
\end{abstract}

Key Words: Gliofibroma; Bimorphic neoplasm; Desmoplastic glioma; Adult population; Tumour suppressor protein 53
Analogous to the mixed epithelial/mesenchymal tumours seen in Müllerian derivatives, some glial neoplasms can display a bimorphic appearance due to an accompanying mesodermal component. While gliosarcoma is probably the best-known paragon of this group, the existence of non-malignant mesenchymal constituents, different from the so-called Scherer's tertiary structures, is rare but feasible. Here, we describe a peculiar case of a highgrade astrocytic neoplasm (glioblastoma) blending with an innocuous spindle-cell component.

\section{CASE REPORT}

A 50-year-old female presented with headache and loss of memory over the previous six months. Magnetic resonance imaging scans showed a left parieto-temporal mass $(70.82 \times 48.56$ $\times 43.97 \mathrm{~mm}$ ) with significant perilesional oedema and postcontrast annular enhancement (Fig. 1A, B). Despite prompt tu- mour sampling and analysis, the patient ultimately expired one month after the surgery. Necropsy was not authorized.

Histologically, the lesion showed areas unequivocally consistent with glioblastoma due to their microvascular proliferation, palisading necrosis, hypercellularity, pleomorphism, and high mitotic activity (Fig. 1C, D). These areas intermingled with cytologically bland, long spindle-cell fascicles (Fig. 1E, F) enveloped by sturdy collagen and fine reticulin fibers (Fig. 2A-D). As expected, immunoperoxidase-coupled reactions showed strong and diffuse expression of glial fibrillary acidic protein in the glioblastoma areas (Fig. 3A), while the spindled part of the neoplasm was exclusively positive for vimentin (Fig. 3B). Ki-67 expression was variable, with positivity as high as $50 \%$ in the astroglial regions (Fig. 3C) but with immunolabeling of less than $1 \%$ in the mesenchymal portion. Likewise, expression of p53 was largely diffuse in the gliomatous parts, albeit focally in some fusiform cells (Fig. 3D, E). No reaction was detected for epidermal growth 

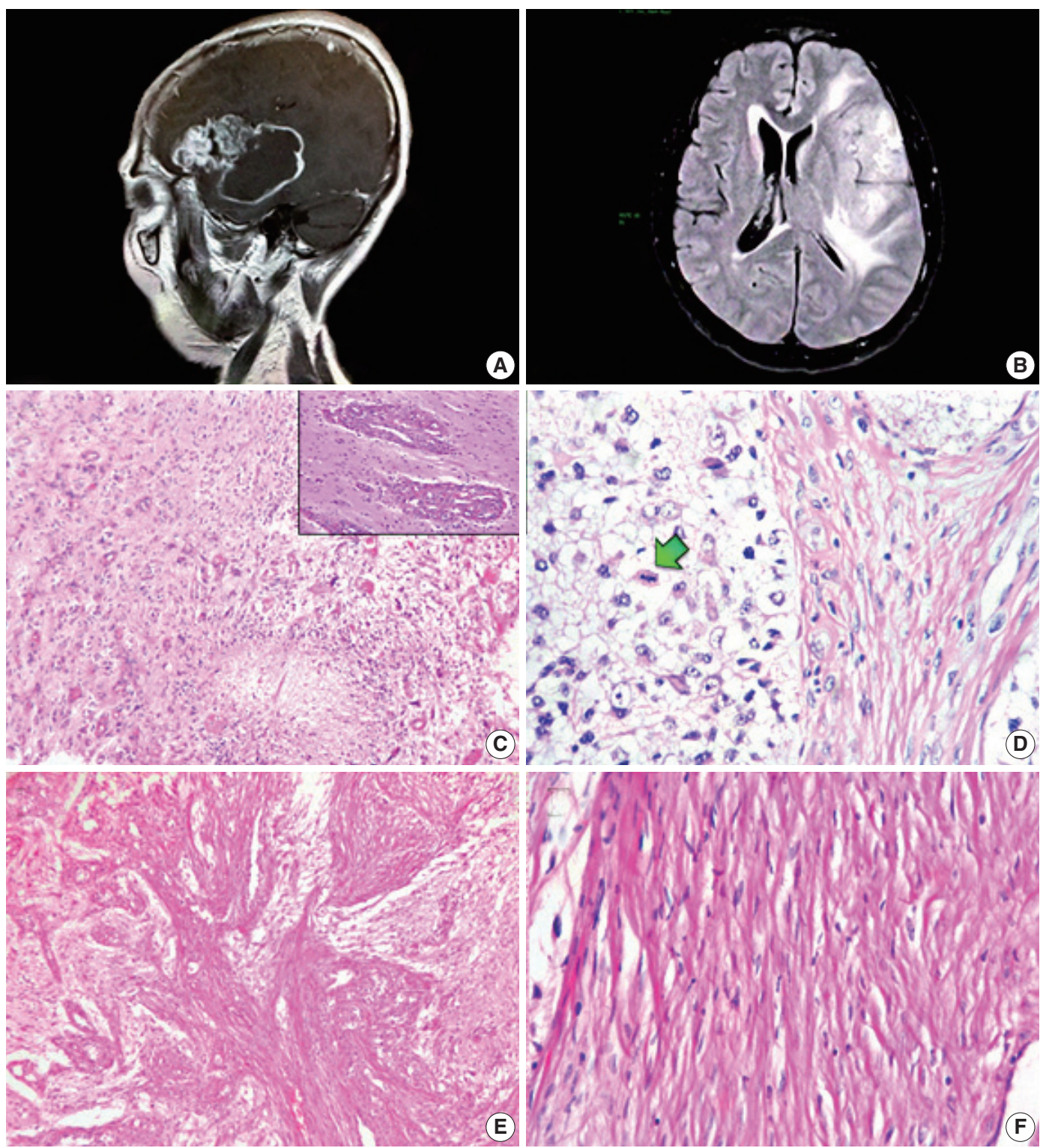

Fig. 1. Magnetic resonance imaging scans and biphasic histological features. (A) Post-contrast, T1-weighted sagittal section. A heterogeneous tumour with an enhancing peripheral rim is shown. (B) Fluid-attenuated inversion recovery sequence. Considerable outlying oedema can be seen. (C) Glioblastomatous component with palisading necrosis and microvascular proliferation (upper right inset). (D) Boundary zone with neoplastic cells of gemistocytic appearance (left) next to apparently atypical spindle cells (right). Mitoses are noticeable exclusively in neoplastic glial cells (arrow). (E) Mesenchymal component with solid fascicular tissue intermingled with loose astrocytic areas. (F) High-magnification photomicrograph of the cytologically bland mesenchymal constituent.

factor receptor in either element.

The lesion was diagnosed as gliofibroma (mixed astrocytic neoplasm with a low-grade mesenchymal constituent), with special note of its poor prognosis based on the degree of anaplasia of the glial component, suggestive of glioblastoma. This note was written prior to the patient's demise.

All procedures performed in this study were in accordance with the ethical standards of the institutional and/or national research committee and with the 1964 Helsinki Declaration and its later amendments or comparable ethical standards. Informed consent was obtained from the patient's legal guardian, and the anonymity of the patient was preserved. 

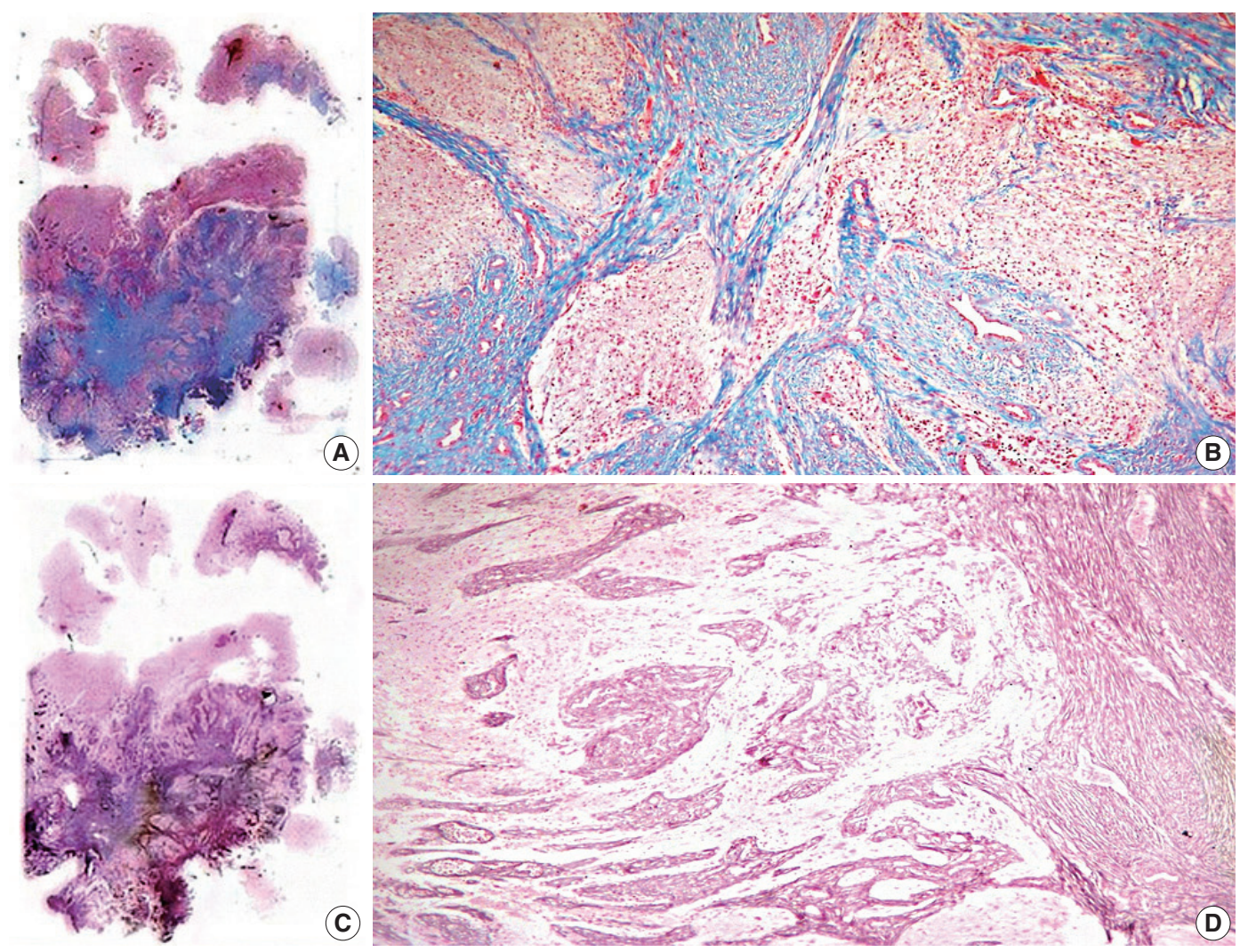

Fig. 2. Histochemical stains of whole-mount sections. (A, B) Collagen-rich tissue seen with Masson's trichrome. (C, D) Reticulin pattern showed with reticular fiber stain.
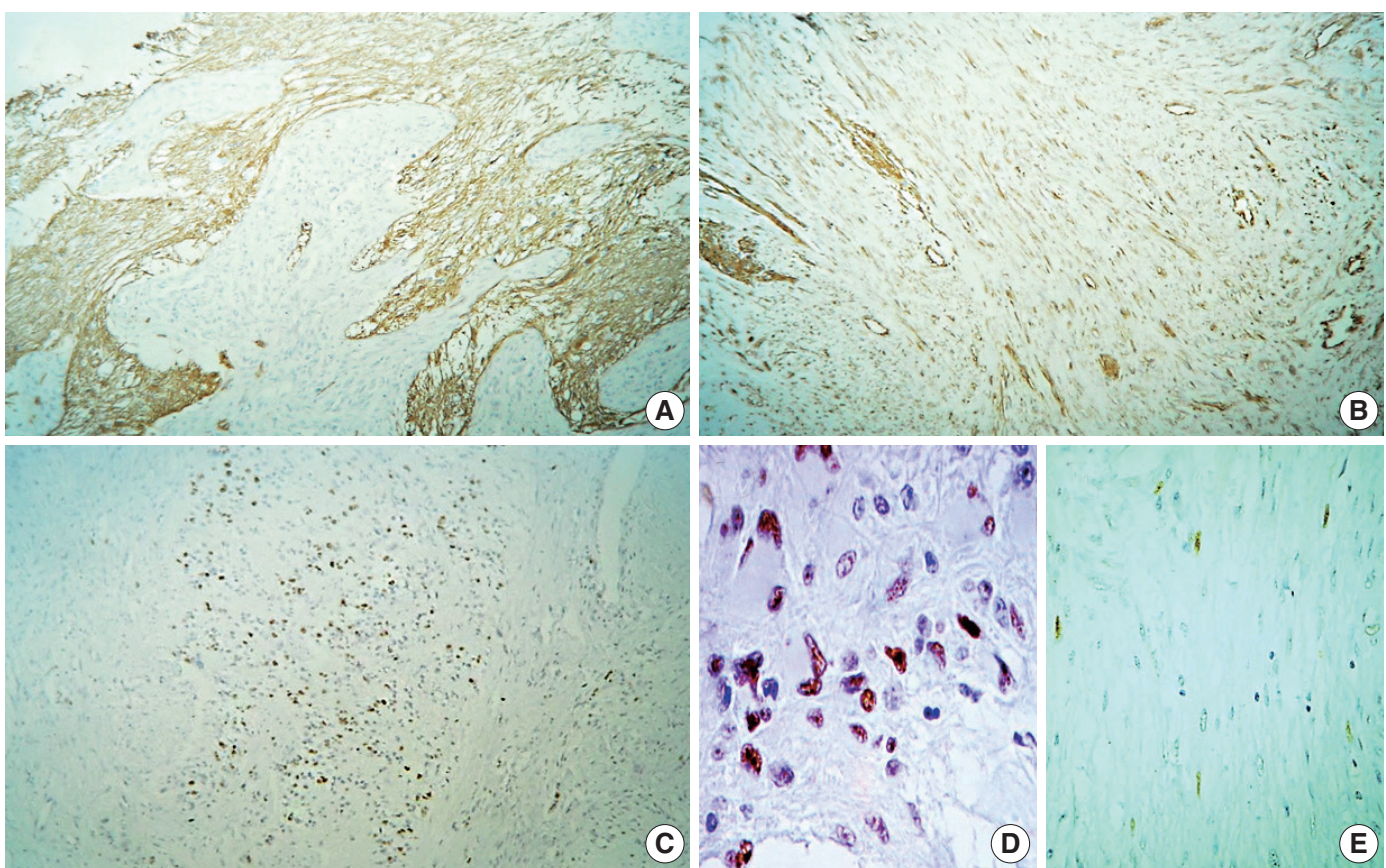

Fig. 3. Immunohistochemistry panel. (A) Glial fibrillary acidic protein. (B) Vimentin. (C) Ki-67. (D) p53 (glial portion) with diffuse immunolabeling. (E) p53 (mesenchymal portion) with very focally positive immunoreactivity. 
Table 1. High-grade gliofibroma cases

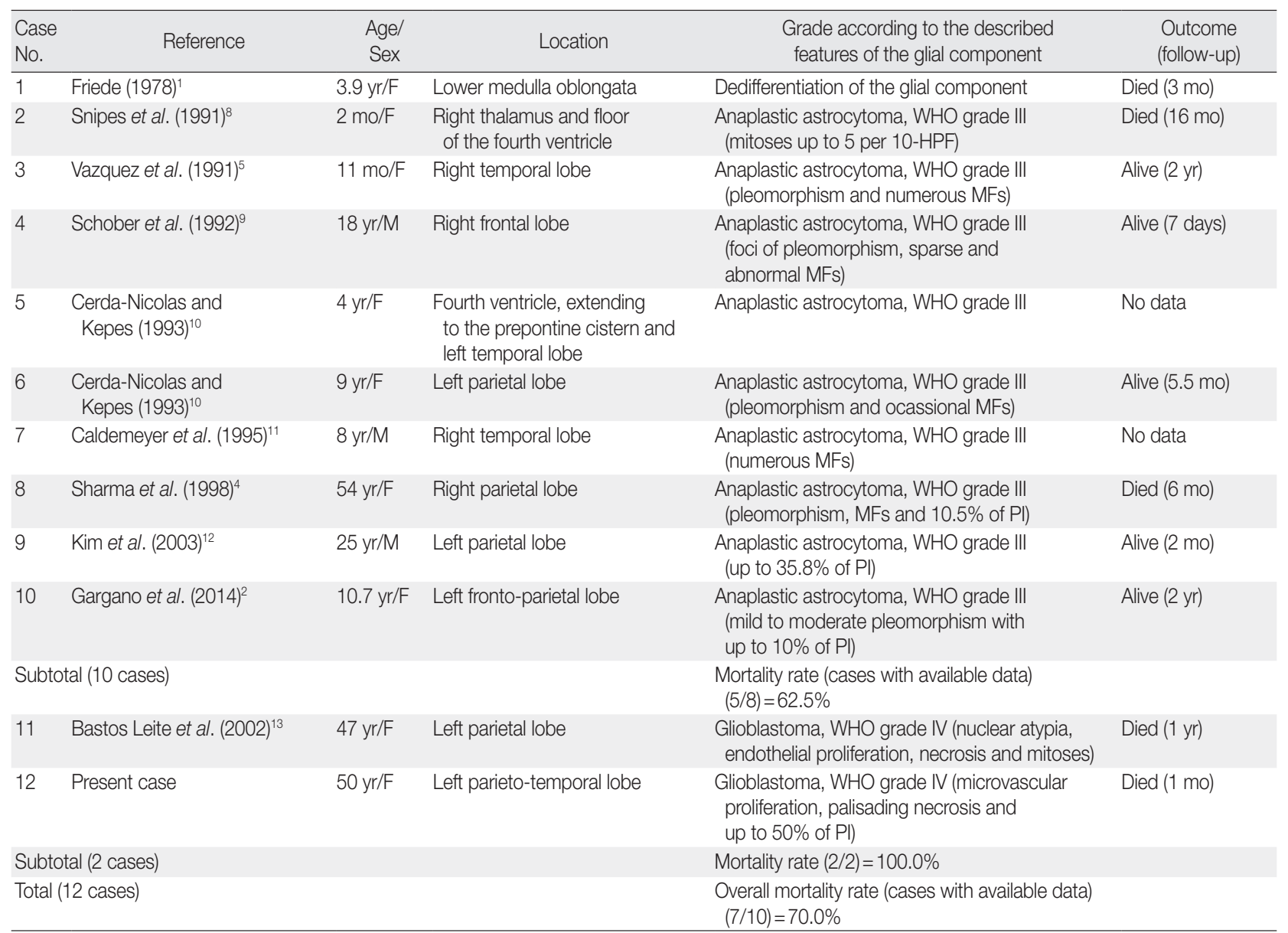

F, female; WHO, World Health Organization; HPF, high-power fields; M, male; MFs, mitotic figures; PI, proliferation index (MIB-1, Ki-67).

\section{DISCUSSION}

The term gliofibroma was first introduced by Friede in $1978^{1}$ to describe an unusual neoplasm featuring astroglial cells within collagen bundles. Since then, nearly 40 cases of gliofibroma have been reported in the worldwide literature, the vast majority occurring in the paediatric population. ${ }^{2}$ Only 11 cases have been recorded in adults, with age ranging from 19 to 54 years., ${ }^{3,4}$ More importantly, some cases described in both, children and adults, bear morphological traits indicative of biologically aggressive lesions (Table 1). The prognostic value of these features in gliofibromas has been widely debated, as some non-lethal tumours have a high-grade glial component while other are recurrent lesions despite their low-grade glial associate. ${ }^{5,6}$ Moreover, as accurately demonstrated by Suarez et al., ${ }^{7}$ the overall mortality for these lesions is approximately $23 \%$. However, when considering the lethality of the high-grade-only subset, this value increases to $70 \%$ or even $100 \%$ in cases with an accompanying gl- ioblastoma component (Table 1). ${ }^{8-13}$

In their work, Schober et al. ${ }^{9}$ were the first to tentatively assign a World Health Organization (WHO) grade to their diagnosis, while Cerda-Nicolas and Kepes ${ }^{10}$ preferred to add a descriptive modifier (anaplastic). In a similar way, Sharma et $a l^{4}{ }^{4}$ proposed to dichotomize these lesions into low-grade (benign) or high-grade (malignant/anaplastic) based on the Ki-67 proliferation index. In spite of these grading attempts, Snipes et al. ${ }^{8}$ seminally pointed out that prognosis seems to be more related to location rather than to any morphological or immunohistochemical feature, though the degree of anaplasia and the mitotic index might be worthy of consideration. On the other hand, the case documented by Altamirano et al..$^{14}$ highlights the issue of whether atypia can exist in the fibroma portion of the neoplasm. This is exceedingly difficult to assert, as the boundaries of either a gliosarcoma or sarcoglioma are unavoidably blurred. In the aforementioned case, increased immunolabeling for $\mathrm{Ki}-67$ was noted in the nuclei of the mesenchymal part, though it was rated 
in less than $5 \%$ of the overall tumour. The authors interpreted the mass as a proliferative activity of uncertain relevance. Though our case displays focal nuclear enlargement and vacuolization (Fig. 1D), we did not observe an increment in mesenchymal Ki-67 expression and did not find any areas suggestive of a true sarcomatous component. Nonetheless, this presumed "atypia" might not reliably worsen the prognosis, as in our case it was based on histology (glioblastoma) while in Altamirano's group was determined by location (thalamus/mesencephalon). p53 immunostaining in our case was also of conjectural significance, indicating not only a possible secondary glioblastoma, but also a common origin of both the glial and mesenchymal elements, similar to the demonstration of Biernat regarding gliosarcomas, ${ }^{15}$ and thus possibly representing a true benign mesodermal differentiation as formerly hypothesised by Snipes et al. ${ }^{8}$ Furthermore, p53 immunolabeling has been previously tested in nine gliofibroma cases with no positive result. , $4,6,12,16-18$ Interestingly, Goyal et al. ${ }^{19}$ also applied this immunohistochemical marker in three other cases but never mentioned the final outcome. This lack of p 53 expression is shared by the so-called desmoplastic infantile astrocytoma and may hint, at least in these aforementioned cases, at a different developmental pathway from that of diffuse or anaplastic astrocytomas and glioblastomas.

Three more issues briefly deserve our attention. (1) As previously mentioned, the first report of gliofibroma clearly detailed a desmoplastic astrocytoma (Friede-type gliofibroma); ${ }^{1}$ nonetheless, this designation was subsequently applied to similar lesions consisting of both glial and mesenchymal derivatives (authentic glioma/fibroma), ${ }^{10}$ as in the present case. It is worth mentioning that the glial constituent can range from innocuous to evidently malignant, while the latter is a consistently benign fusocellular component. (2) In the bimorphic subset, it has not been fully corroborated whether the two populations are distinct cell lineages or if the fusiform cells are glia capable of differentiating into fibroblasts, myofibroblasts, Schwann cells or histiocytes. ${ }^{2,20}$ (3) Recently, some desmoplastic lesions displaying a non-astrocytic glial phenotype with immunohistochemical properties more akin to ependymoma (as in the case described by Gargano et al. ${ }^{2}$ ) have also been diagnosed as gliofibromas. Thus, as pointed out by Suárez et al., a controversial dilemma of "splitting versus lumping" currently prevails in the conceptual and classification schemes regarding these kinds of tumours. Nevertheless, it seems to be more of an academic and histopathological predicament rather than one of clinical concern or prognostic relevance.

As shown in our case and previously advised by other authors, gliofibroma prognosis is greatly influenced by the degree of an- aplasia of the glial component. Hence, we suggest adding a grade (WHO I-IV) or descriptive (low-grade, high-grade, anaplastic, malignant/glioblastomatoid) modifier to the diagnosis of gliofibroma.

It had been previously claimed that necrosis and prominent vascular proliferation are not usual features of gliofibromas. ${ }^{16}$ Thus, we document the second case of gliofibroma with a glioblastomatous component ${ }^{13}$ (though this is the first case involving a mixture of glioma and fibroma) as well as the third case occurring in Latin American population. ${ }^{2,14}$

\section{Conflicts of Interest}

No potential conflict of interest relevant to this article was reported.

\section{REFERENCES}

1. Friede RL. Gliofibroma: a peculiar neoplasia of collagen forming glia-like cells. J Neuropathol Exp Neurol 1978; 37: 300-13.

2. Gargano P, Zuccaro G, Lubieniecki F. Intracranial gliofibroma: a case report and review of the literature. Case Rep Pathol 2014; 2014: 165025.

3. Prayson RA. Disseminated spinal cord astrocytoma with features of gliofibroma: a review of the literature. Clin Neuropathol 2013; 32: 298-302.

4. Sharma MC, Gaikwad S, Mehta VS, Dhar J, Sarkar C. Gliofibroma: mixed glial and mesenchymal tumour: report of three cases. Clin Neurol Neurosurg 1998; 100: 153-9.

5. Vazquez M, Miller DC, Epstein F, Allen JC, Budzilovich GN. Glioneurofibroma: renaming the pediatric "gliofibroma": a neoplasm composed of Schwann cells and astrocytes. Mod Pathol 1991; 4: 519-23.

6. Deb P, Sarkar C, Garg A, Singh VP, Kale SS, Sharma MC. Intracranial gliofibroma mimicking a meningioma: a case report and review of literature. Clin Neurol Neurosurg 2006; 108: 178-86.

7. Suarez CR, Raj AB, Bertolone SJ, Coventry S. Carboplatinum and vincristine chemotherapy for central nervous system gliofibroma: case report and review of the literature. J Pediatr Hematol Oncol 2004; 26: 756-60.

8. Snipes GJ, Steinberg GK, Lane B, Horoupian DS. Gliofibroma: case report. J Neurosurg 1991; 75: 642-6.

9. Schober R, Bayindir C, Canbolat A, Urich H, Wechsler W. Gliofibroma: immunohistochemical analysis. Acta Neuropathol 1992; 83 : 207-10.

10. Cerda-Nicolas M, Kepes JJ. Gliofibromas (including malignant forms), and gliosarcomas: a comparative study and review of the 
literature. Acta Neuropathol 1993; 85: 349-61.

11. Caldemeyer KS, Zimmerman RA, Azzarelli B, Smith RR, Moran CC. Gliofibroma: CT and MRI. Neuroradiology 1995; 37: 481-5.

12. Kim Y, Suh YL, Sung C, Hong SC. Gliofibroma: a case report and review of the literature. J Korean Med Sci 2003; 18: 625-9.

13. Bastos Leite AJ, Palmeira de Sousa C, Honavar M. Malignant gliofibroma (desmoplastic glioblastoma) in an adult: case report. J Neuroradiol 2002; 29: 215-8.

14. Altamirano E, Jones MC, Drut R. Gliofibroma. Comunicación de un caso pediátrico y revisión de la bibliografía. Patol Rev Latinoam 2011; 49: 221-5.

15. Biernat W, Aguzzi A, Sure U, Grant JW, Kleihues P, Hegi ME. Identical mutations of the $\mathrm{p} 53$ tumor suppressor gene in the gliomatous and the sarcomatous components of gliosarcomas suggest a common origin from glial cells. J Neuropathol Exp Neurol 1995; 54: 651-6.

16. Prayson RA. Gliofibroma: a distinct entity or a subtype of desmoplastic astrocytoma? Hum Pathol 1996; 27: 610-3.

17. Kim NR, Suh YL, Shin HJ, Park IS. Gliofibroma with extensive calcified deposits. Clin Neuropathol 2003; 22: 14-22.

18. Matsumura A, Takano S, Nagata M, Anno I, Nose T. Cervical intramedullary gliofibroma in a child: a case report and review of the literature. Pediatr Neurosurg 2002; 36: 105-10.

19. Goyal S, Puri T, Gunabushanam G, et al. Gliofibroma: a report of three cases and review of literature. Acta Oncol 2007; 46: 1202-4.

20. Erguvan-Onal R, Ateş O, Onal C, Aydin NE, Koçak A. Gliofibroma: an incompletely characterized tumor. Tumori 2004; 90: 157-60. 